\title{
Boltay Hath for Indian Sign Language Recognition
}

\author{
Sarbjeet Kaur ${ }^{1}$ \\ PTU Jalandhar \\ Amritsar college of engineering and technology \\ Amritsar, India
}

\author{
V. K. Banga ${ }^{2}$, Ph.D \\ PTU Jalandhar \\ Amritsar college of engineering and technology \\ Amritsar, India
}

\begin{abstract}
In this research work it is discussed that how a gesture recognition system for alphabetical hand gestures is built. The main motive was to develop a system that can simplify the way that the normal human being interact with the deaf and dump people without the need of interpretator. This system also provides the easy way of humans interaction with computers. This system is designed using MATLAB instead of neural network. In this system Eigen vector and Eigen value techniques only in MATLAB are used. The basis of our method is a fast detection process to obtain the meaningful hand region from the whole image, which is able to deal with a large number of hand gestures against different indoor backgrounds and lighting condition, and a recognition process that identifies the hand gestures from the images of the normalized hand. Experiments show that the system can achieve a $99 \%$ recognition rate and is suitable for real-time applications.
\end{abstract}

\section{Keywords}

Hand gestures, human computer interaction, Graphical user interface, MATLAB.

\section{INTRODUCTION}

The hand movement, body movement and facial expression can also used for conveying the information i.e. it also called manual communication. This type of communication is mostly used in deaf dump community. If one person is normal and verbally communicate with other but the other person is deaf and dump and not communicate verbally with the first person then he needs an interpretator which provide a easy way of communication between the deaf - dump people and normal person. Hand gestures can help us say more in less time. If a deaf- dump person made a gesture to communicate with a normal person that gestures is named a Boltay hath. In this paper, used only hand movement for making a hand poster or hand gesture. In this paper, make a gesture without using any types of gloves and is not used any type of marker . Hand poster are two type first is static and second is dynamic type. In this paper, only used static hand movement image or hand gesture. Some gestures also have both static and dynamic elements, as in sign languages. In this work only on one hand gesture is used instead of two hands but most of the researcher's uses both hands. The data is analysis from an instrumented data for use in recognition of some signs and gestures. A system is developed for recognizing these signs and their conversion into alphabets (words). In this research work it is tried to make a system for Indian sign language.

\section{RELATED WORK}

Almost all the researchers have used neural network but in this paper only MATLAB is used instead of any other tool. To improve the interaction in qualitative terms in dynamic environment it is desired that the means of interaction should be as ordinary and natural as possible. These movements may include the simple action of pointing by finger to more complex ones that are used for communication among people[5]. Aseema Sultana, T Rajapuspha [15] provides a hand gesture recognitions system using SVM classifier. In this paper it also uses a Haar Wavelet Transformation techniques. According to Aseema Sultana, T Rajapuspha its final stage is stage is hand gesture recognition in which the output of current gesture model from the second stage is compared with each model in hand gesture database where the most matched hand gesture is selected as final recognition result. Here the Support Vector Machine (SVM) Classifier is used for statistical classification and regression analysis. G. R. S. Murthy \& R. S. Jadon[5] presents a Model based Approaches (Kinematic Model) attempt to infer the pose of the palm and the joint angles. Such an approach would be ideal for realistic interactions in virtual environments. Generally, the approach consists of searching for the kinematic parameters that brings the $2 \mathrm{D}$ projection of a $3 \mathrm{D}$ model of hand into correspondence with an edge-based image of a hand. Machine Learning based on Hidden Markov Models (HMMs). Cristina Manresa, Javier Varona, Ramon Mas and Francisco J. Perales[1], presents a techniques based on the probability that a new pixel of skin can be calculated. To prevent errors from hand segmentation the second step is hand tracking. Tracking is performed assuming a constant velocity model and using a pixel labeling approach. From the tracking process several hand features are extracted that are fed to a finite state classifier which identifies the hand configuration. Prof. K.Rama Linga Reddy, G.R. Babu, and Dr. Lal Kishore [10] present a paper based on face recognition using artificial neural network. The Principal Component Analysis (PCA) or Linear Discriminant Analysis (LDA) method is employed. The weight vector of face images to be trained become the input to neural network classifier. The proposed algorithm has been tested on 400 faces of 10 subjects of ORL data base and 500 faces of 100 subjects of FERET database results are encouraging compared to the existing methods. Satonkar Suhas S, Kurhe Ajay B, Dr. Prakash Khanale B [14] work on Holistic approach for face recognition. The success rate of classification of images is $100 \%$. The value of projection vectors is $0.0076,0.0056$, $0.0008,0.0036$ and 0.0028 . K M Poornima, Ajit Danti, and S K Narasimhamurthy [21] present a paper for face recognition. In this paper, it uses discrete wavelet transform (DWT), independent component analysis (ICA) and classification using k-Nearest Neighborhood (k-NN) classifier. This system obtain a recognition rate of about $83.5 \%$. Simarnjot singh and Navneet kaur [22] provides a hand recognition system based on SIFT ( Scale invariant feature transform ) algorithm. In this paper, the system detect hand shape contour region and find the maximum contour according to skin color and angle of the finger position. Arathy.V and Dr.P.Srinivasa Babu[24] provides a system for recognition of face images through the 
provides a system for recognition of face images through the Fusion Approach. In this it uses Gabor filter and Principal component analysis (PCA) is a dimensionality reduction technique which is used for compression and face recognition problems. The hand can be classified into one of the four gesture classes or one of the four different movement directions. Finally, using the system's performance evaluation result the usability of the algorithm in a videogame environment is shown.

\section{SYSTEM ARCHITECTURE}

The system architecture of hand gestures is given below. According to system architecture first needs a signer which made a hand sign in front of the web camera. Capture these hand signs and stored in data base. In the proposed method, 32 combinations of binary images each representing_UP' and =DOWN' position of 5 fingers shown in the Fig 1:-first, All the hand gesture images are captured and stored these hand poster images in dataset. 200 hand gestures are stored in dataset folder. This system architecture divide into four parts :-
A. Modules for changing the size of the gesture.
B. Preprocessing module for gesture.
C. Mean image.
D. $\mathrm{O} / \mathrm{p}$ result.

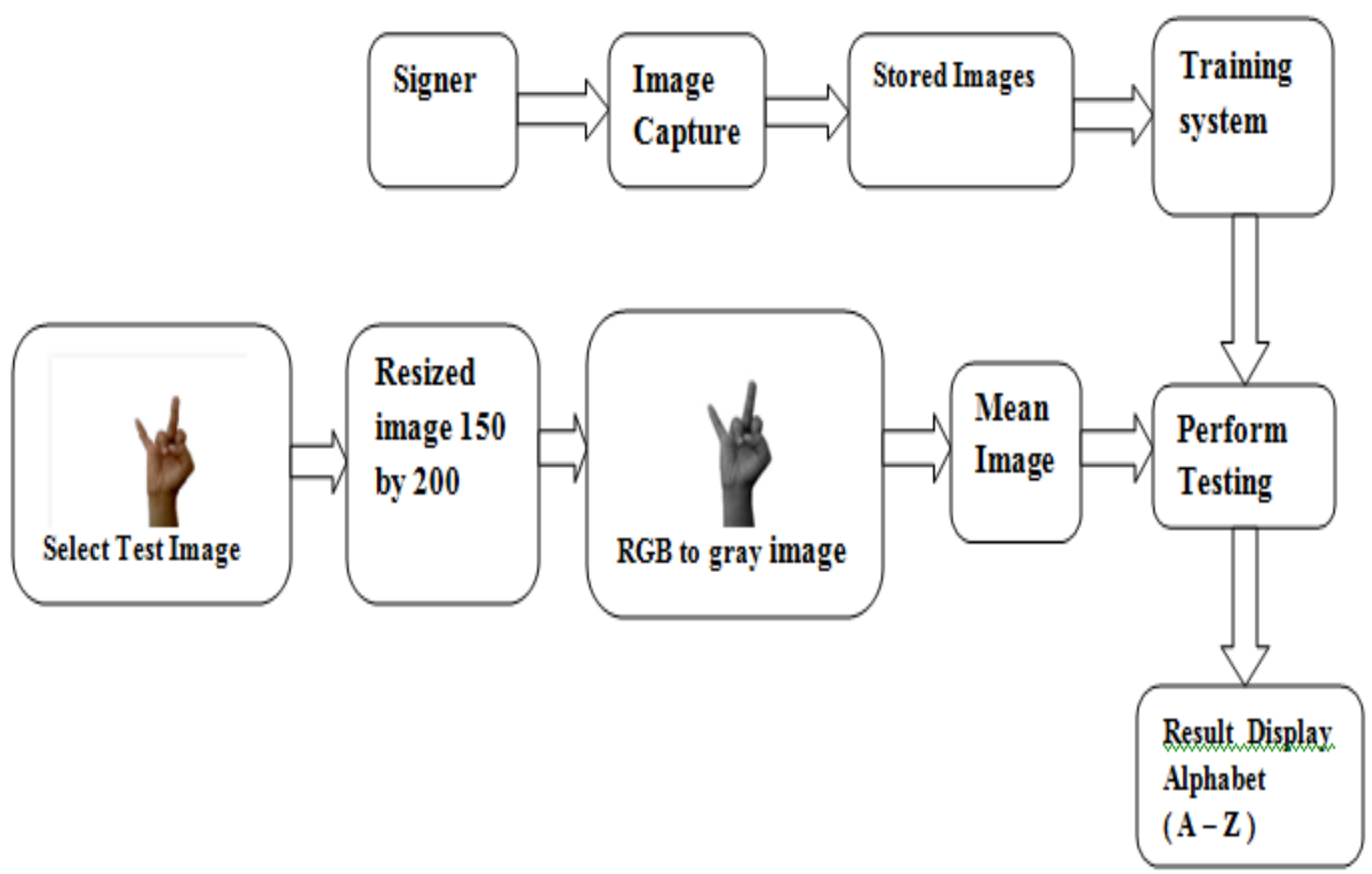

Fig 1: Shows the system architecture

\subsection{Modules for Changing the size of the gesture}

In this section firstly select the test image from the database images. Then change the size of the image into $1024 x 768$ bye $160 x$ 120. It takes the color image and the convert the color image into gray scale image i.e. 0 and 1 in binary form. After that it converts the data type of image to double calculation.

\subsection{Preprocessing module for gesture}

In this section, convert the resized image into black and white image. If the RGB image is 24-bit, each channel has 8 bits, for red, green, and blue - in other words, the image is composed of three images (one for each channel), where each image can store discrete pixels with conventional brightness intensities between 0 and 255. The RGB color space (Red, Green and Blue which considered the primary colors of the visible light spectrum) is converted through gray scale image to a binary image. Many images come in color and certain operations in MATLAB are only defined on grayscale Images. Use the function rgb2gray to covert them to grayscale.

\subsection{Mean image}

In this section, the important part is feature extraction. The importance of feature extraction phase is to know the meaning 


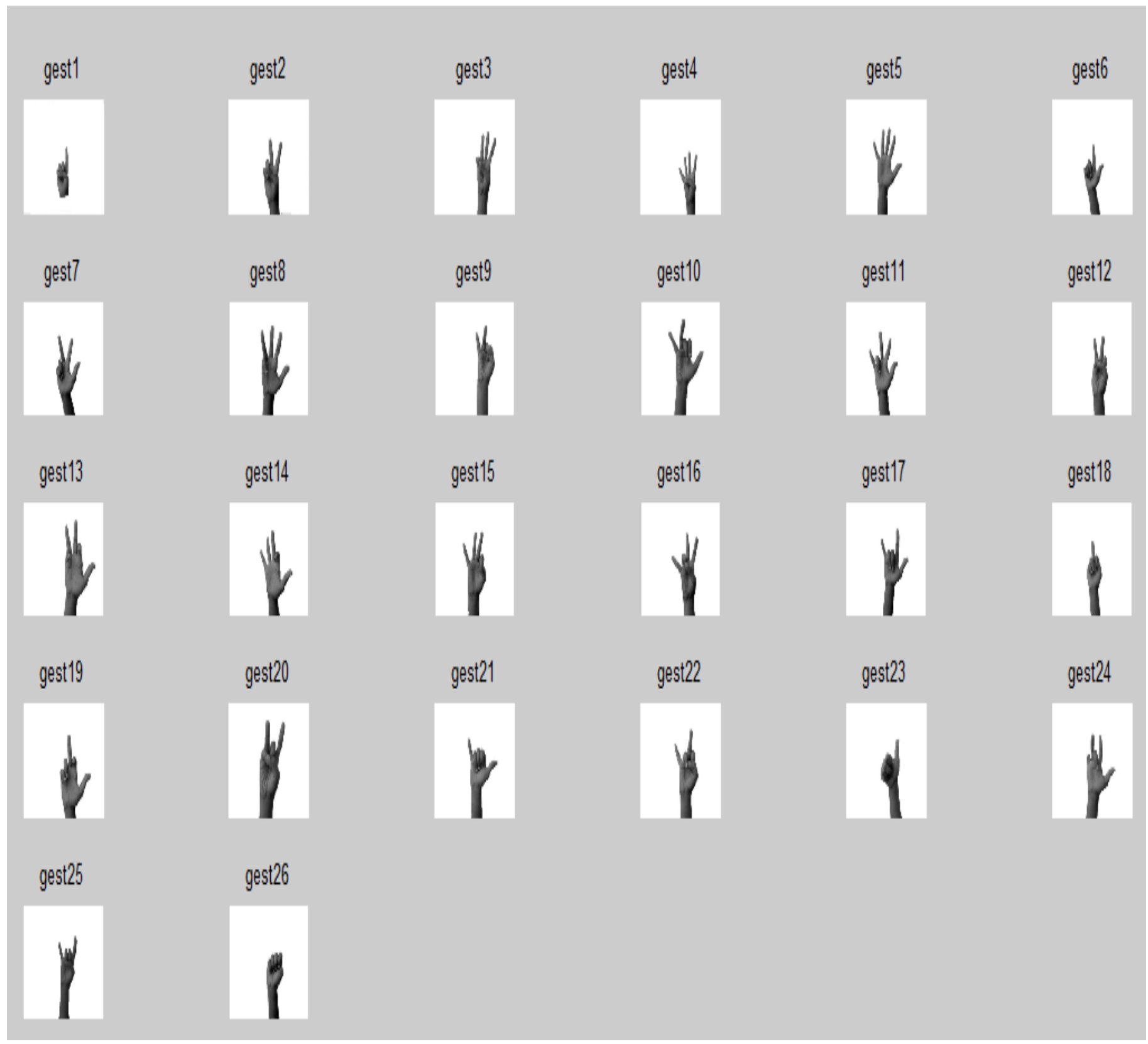

Fig 2:- Background gray scale dataset hand gesture.

of the letters and accordingly to understand the signed word. Selecting the right set of features is the decisive key in order to avoid ambiguity in all pattern recognition systems. Transforming the input data into the set of features is called feature extraction. If the features extracted are carefully chosen it is expected that the features set will extract the relevant information from the input data in order to perform the desired task using this reduced representation instead of the full size input[9].

For $\mathbf{B}$, an $\boldsymbol{N} \times \boldsymbol{N}$ matrix the real number is called an Eigen value of $\mathbf{S}$ if there exists a nonzero vector $\boldsymbol{x}$ in $\mathbf{R} \boldsymbol{\eta}$ such that $\boldsymbol{B} \boldsymbol{x}=\boldsymbol{x}$. The vector is called an eigenvector $\boldsymbol{x}$. The equation $\boldsymbol{B} \boldsymbol{x}=\boldsymbol{x}$ is equivalent to $(\boldsymbol{B}-\boldsymbol{I}) \boldsymbol{x}=\mathbf{0}$, so all of the following are equivalent:-
2. $(\boldsymbol{B}-\boldsymbol{I}) \boldsymbol{x}=\mathbf{0}$ has a nontrivial solution.

3. $\boldsymbol{B}-\boldsymbol{I}$ is singular.

4. $\operatorname{det}(B-I) x=0$.

The eigenvectors for are the nonzero solutions $\boldsymbol{x}$ to $(\boldsymbol{B}-\boldsymbol{I}) \boldsymbol{x}=\mathbf{0}$. These vectors together with the $\mathbf{0}$ vector is called the Eigen space corresponding to Eigen value. The expression det (B-I) is a polynomial in of degree $\boldsymbol{N}$, called the characteristic polynomial. By property 4 , the Eigen values are the roots of the characteristic equation $\operatorname{det}(B-I) x=0$.

1. $\boldsymbol{N} \times \boldsymbol{N}$ is an Eigen value of $\mathbf{B}$. 
Determining Eigen values and eigenvectors with MATLAB:-

In MATLAB , the characteristic polynomial of a matrix $\mathbf{A}$ is found by entering $\operatorname{poly}(B)$. If $A$ is an $N \times N$ matrix, $\operatorname{poly}(B)$ is a row vector with $\boldsymbol{N}+\mathbf{1}$ elements that are the coefficients of the characteristic polynomial. The command $\operatorname{roots}(\mathbf{C})$ computes the roots of the polynomial whose coefficients are the elements of the vector $\mathbf{C}$. Thus, $\operatorname{roots}(\operatorname{poly}(\mathbf{B}))$ returns the Eigen values of $\mathbf{A}$ in a column vector.

To find the eigenvectors corresponding to each Eigen value found above, it needs to find the nonzero solutions $\boldsymbol{x}$ to $(\boldsymbol{B}-\boldsymbol{I}) \boldsymbol{x}=\mathbf{0}$. One way of doing this in MATLAB is to compute $\boldsymbol{r} \boldsymbol{e} \boldsymbol{f}(\boldsymbol{B}-\boldsymbol{I})$ and then use Gauss-Jordan elimination. The simplfied method is to input of MATLAB. A second method of determining the Eigen values and Eigen vectors in MATLAB is to use the Eig function. For an $\boldsymbol{N} \times \boldsymbol{N}$ matrix B, $\boldsymbol{e i g}(\boldsymbol{B})$ returns a $\boldsymbol{N} \times \mathbf{1}$ column vector whose elements are the Eigen values of $\mathrm{B}$. The command in the form.

$$
[V, D]=e i g(B)
$$

computes both the Eigen values and eigenvectors of $\boldsymbol{B} . \boldsymbol{V}$ will be a matrix whose columns are eigenvectors of $\boldsymbol{B}$ and $\boldsymbol{D}$ will be a diagonal matrix whose entries along the diagonal are Eigen values of $\boldsymbol{B}$. The $i$ th column of $\boldsymbol{V}, \boldsymbol{V}(:, \boldsymbol{I})$, is the eigenvector corresponding to the Eigen value $\boldsymbol{D}(\boldsymbol{I}, \boldsymbol{I})$. Here, $\boldsymbol{D}$ is an $\boldsymbol{N} \times \boldsymbol{N}$ matrix with our eigenvalues on its diagonal and $\boldsymbol{V}$ is an $\boldsymbol{N} \times \boldsymbol{N}$ matrix with columns that represent Eigen vectors. So, their values satisfy the equation:-

$$
\boldsymbol{B} * \boldsymbol{V}=\boldsymbol{V} * \boldsymbol{D}
$$

Or if one wants to look at one Eigen value / Eigen vector at a time:-

$$
B * V(I,:)=D(I, I) * V(I,:)
$$

This stands for $\boldsymbol{A}$ times the $\boldsymbol{I}-\boldsymbol{t} \boldsymbol{h}$ column of $\boldsymbol{V}$ (an eigenvector) equals the $\boldsymbol{I}-\boldsymbol{t h}$ row/ $\boldsymbol{I}-\boldsymbol{t h}$ column entry of $\boldsymbol{D}$ (which of course falls on the diagonal), time the column of $\boldsymbol{V}$. The steps of GUI model for Hand Gesture System are as follows :-

Step 1:- Create a blank big_matrix.

Step 2:- Read the image .

Step 3:- Convert the read color image into RGB2gray scale.

Step 4:- Resized the actual gray scale image.

Step 5:- Now, Subplot the resized image ( Firstly take the square root of columns and square roots of rows ).

Step 6:- Then show the image.

Step 7:- Now Create a singleton matrix i.e.

temp $=$ reshape $($ image I.row $*$ I.coloumn $)$.

Step 8:- Now, substitute the value of temp in step first:-

$$
\text { Blank big }_{\text {matrix }}=\text { temp }
$$

Step 9:- obtain the mean of each row i.e.
Avg $=\operatorname{mean}\left(\right.$ Blank big $\left.{ }_{\text {matrix }}\right)$.

Step 10:- At last when the GUI model of hand gesture recognition system is run then it shows dataset all stored hand gesture images in gray scale.

\section{$3.4 \mathrm{O} / \mathrm{p}$ result}

In this section, this is last step of image recognition sytem. As signing a new feature vector to some predefined categories in order to recognize the sign is the task of classification.

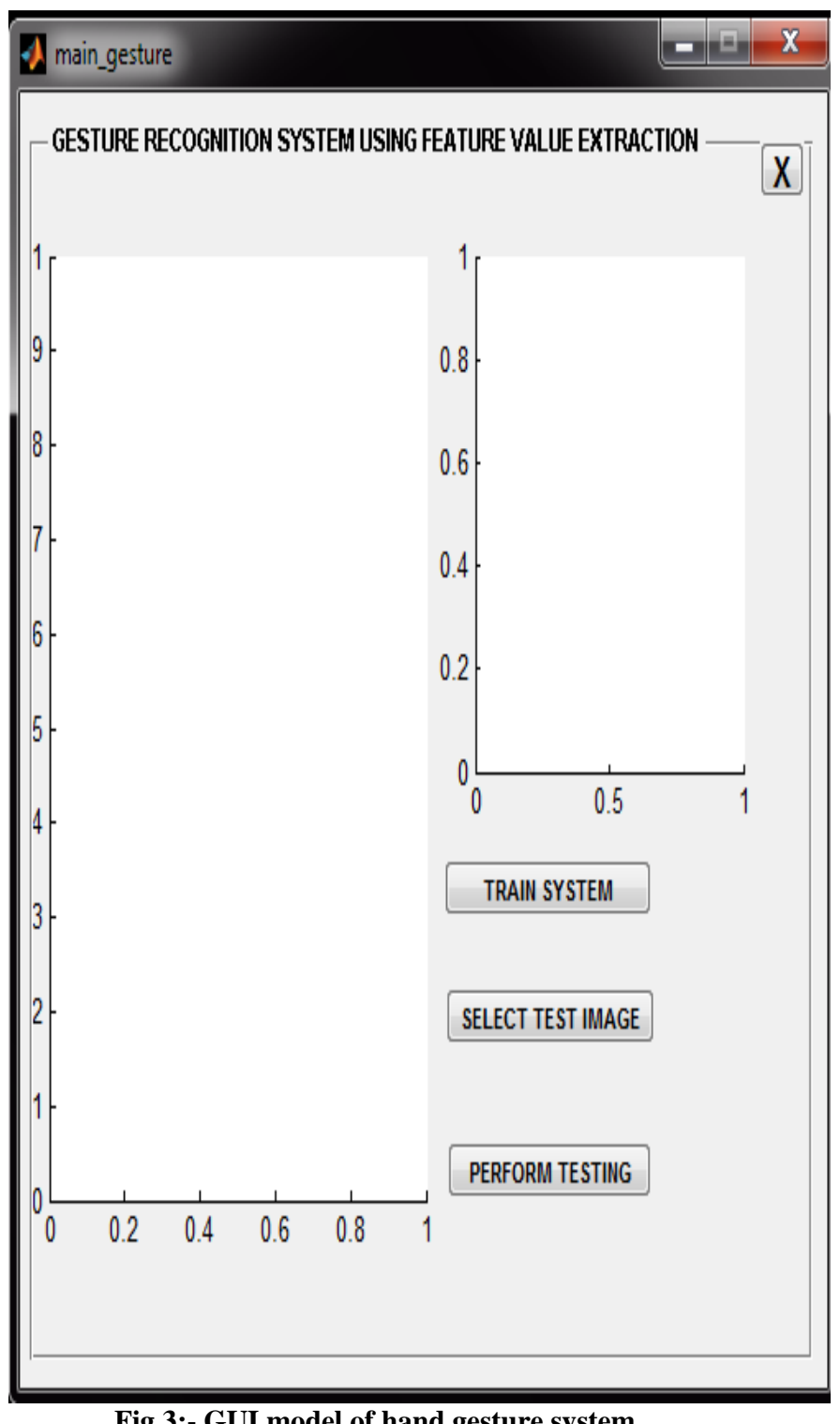

The choice of classification algorithm it is highly depending on the recognition system. A Graphical User Interface (GUI) has been created to automatically train and recognize the gestures as shown in the fig 3 above. 


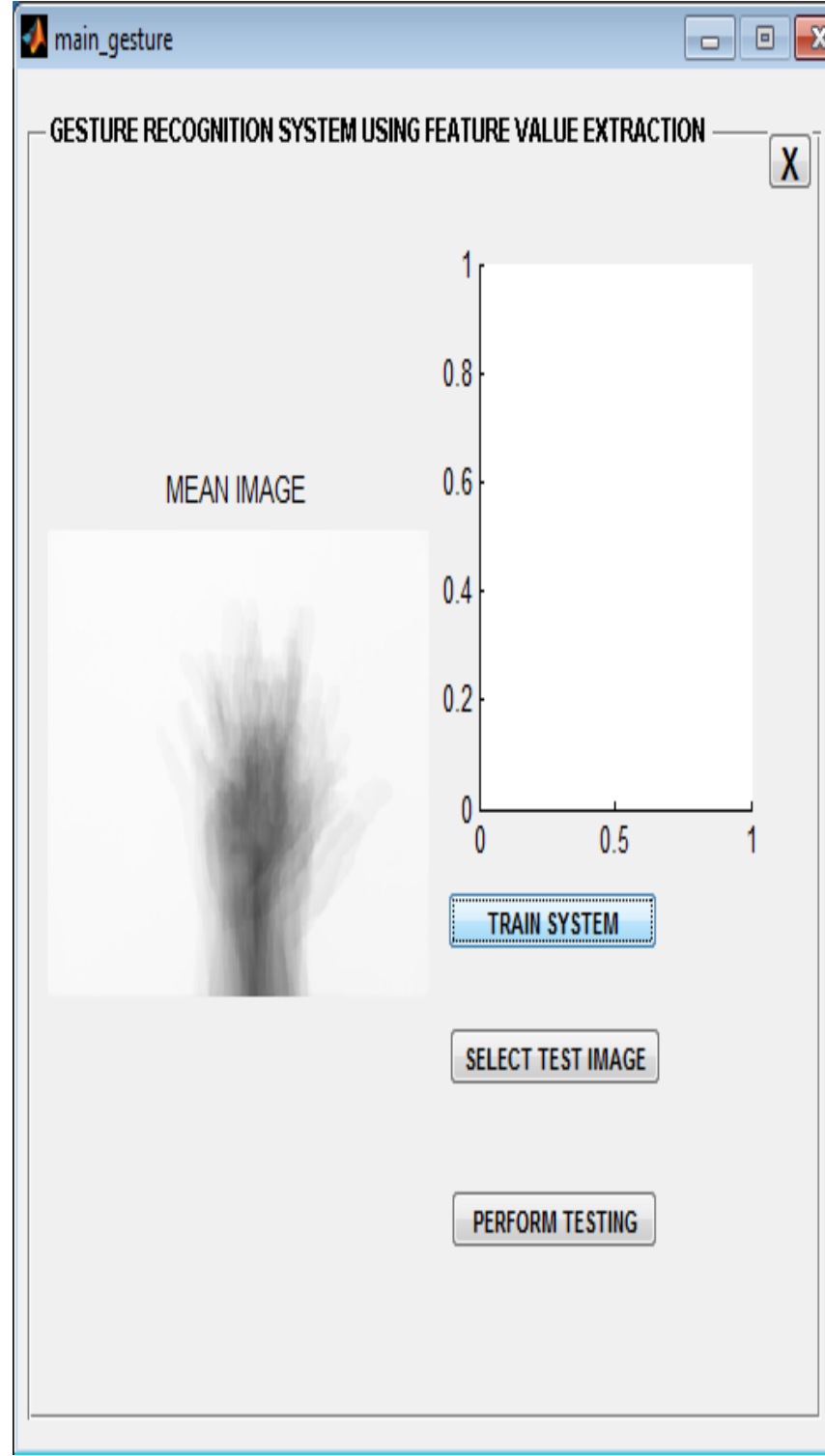

Fig 4:- Example of mean image

In the first step, when the train system button is pressed, it train the system and creates mean image and also gray scale data set in the background. Fig2 and fig4 shows the background gray scale dataset hand gesture images and mean image.

In the second step, when the select test image button is pressed then it provides a folder. In this folder, all the RGB color hand gestures images have stored. Then choose one color hand gesture image amongst them. The hand gesture system resized the chosen image in the background and also convert color image into gray scale image. Gray scale hand gesture image is shown into second axis of the GUI model (Fig5 shows the example of select test image respectively).

In the third step, when the Perform testing button is pressed, then system compares select test image with the mean image and gives the results in alphabetical form.

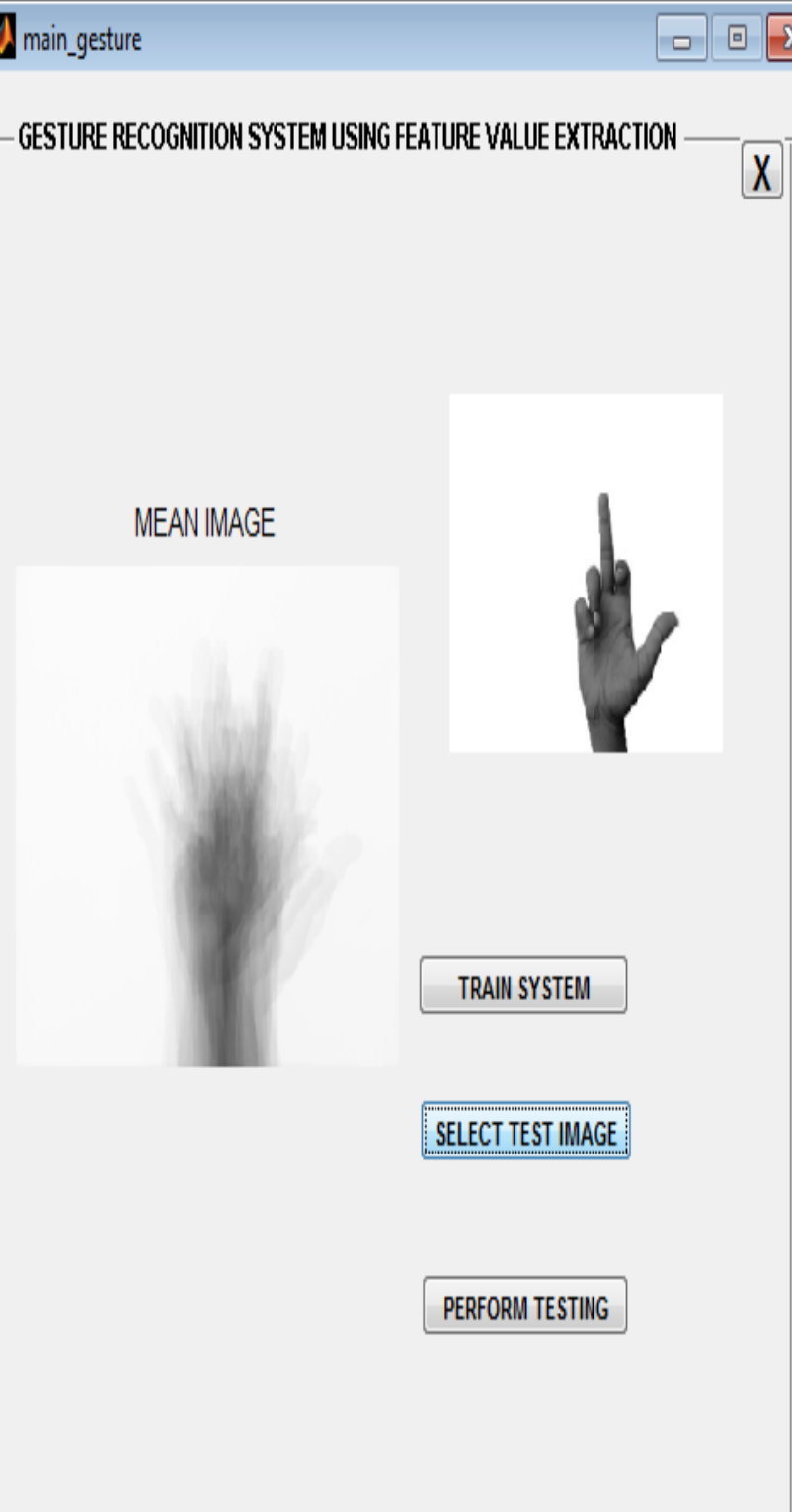

Fig 5:- Example of Select test image.

\section{EXPERIMENTAL RESULT}

This proposed system showed good results to recognize alphabets in real time from RGB color image . Fig6 shows the example of GUI model for hand gesture. The performance of the system is evaluated on its ability to correctly classify samples to their corresponding classes. The recognition rate is defined as the ratio of the number of correctly classified samples to the total number of samples, i.e.

Recognition ratio $=\#$ Recognized Gestures $X 100$

\# Test Gestures 


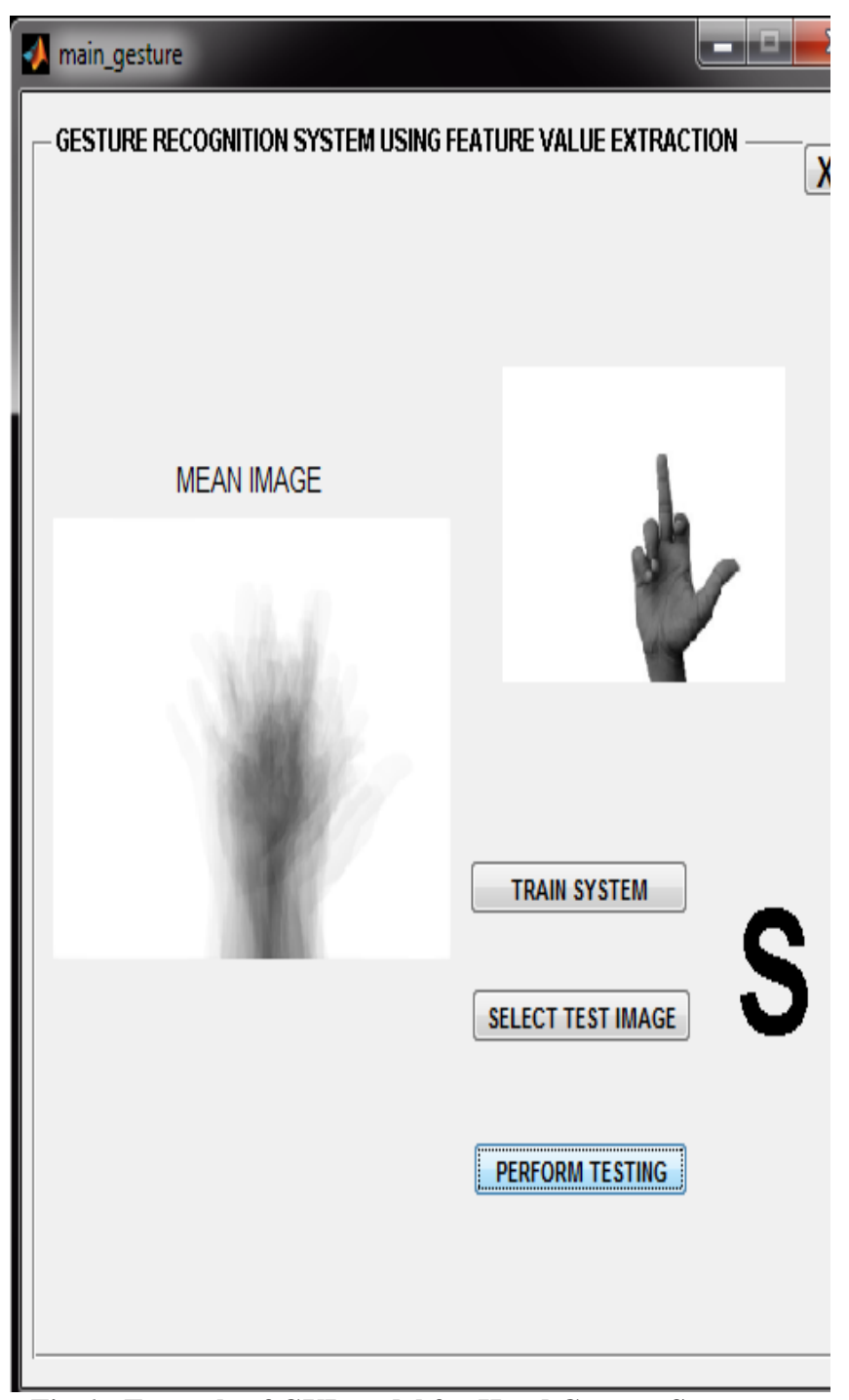

Fig 6:- Example of GUI model for Hand Gesture System

\section{CONCLUSION}

In this project, A system is developed for the purpose of the recognition of a subset of the Indian sign language. system has two phases: the feature extraction phase and the classification phase. The work was accomplished by training a set of input data (feature vectors). Without the need of any gloves, an image for the sign is taken by a web camera. After processing, feature extracting phase depends on Eigen algorithm which is tolerant to gaps in feature boundary descriptions and it is relatively unaffected by image noise. Computer Vision methods for hand gesture interfaces must surpass current performance in terms of robustness and speed to achieve interactivity and usability. The system could be integrated with finger spelling recognition system such as "Boltay Haath" for a complete communication between the common person and the vocally disable people. This reserch works on static hand gesture using only MATLAB. In future the researchers can work on dynamic hand gesture using MATLAB instead of neural network and any other tool.

\section{REFERENCES}

[1] Cristina Manresa, Javier Varona, Ramon Mas and Francisco J. Perales, "Real -Time Hand Tracking and Gesture Recognition for Human-Computer Interaction,
Electronic Letters on Computer Vision and Image Analysis 0(0):1-7, 2000.

[2] Xiaolong Teng, Bian Wu, Weiwei Yu, Chongqing Liu "A hand gesture recognition system based on local linear embedding", X. Teng et al. / Journal of Visual Languages and Computing 16 (2005) 442-454. www.elsevier.com/locate/jvlc.

[3] Yuehui Chen, Shuyan Jiang, Ajith Abraham, "Face Recognition Using DCT and Hybrid Flexible Neural Tree”, 0-7803-9422-4/05/\$20.00 @2005 IEEE.

[4] Noor Saliza Mohd Salleh*,1, Jamilin Jais1, Lucyantie Mazalan1, Roslan Ismail1, Salman Yussof1, Azhana Ahmad1, Adzly Anuar1, Dzulkifli Mohamad", "Sign Language to Voice Recognition: Hand Detection Techniques for Vision-Based Approach", Current Developments in Technology-Assisted Education (2006).

[5] G. R. S. Murthy \& R. S. Jadon, "A REVIEW OF VISION BASED HAND GESTURES RECOGNITION", International Journal of Information Technology and Knowledge Management, JulyDecember 2009, Volume 2, No. 2, pp. 405-410

[6] Pragati Garg, Naveen Aggarwal and Sanjeev Sofat, "Vision Based Hand Gesture Recognition", World Academy of Science, Engineering and Technology 25 2009.

[7] S S Rautaray, A Agrawal, “ A Novel Human Computer Interface Based On Hand Gesture Recognition Using Computer Vision Techniques", in Proceeding of ACM IITM' 10, pp.292-296,2010.

[8] Vaishali S. Kulkarni ${ }^{1}$, Dr. S.D.Lokhande ${ }^{2}$, “Appearance Based Recognition of American Sign Language Using Gesture Segmentation", Vaishali.S.Kulkarni et al. / (IJCSE) International Journal on Computer Science and Engineering, Vol. 02, No. 03, 2010, 560-565.

[9] Xu Zhang, Xiang Chen, Yun Li, Vuokko Lantz, Kongqiao Wang, and Jihai Yang, "A Framework for Hand Gesture Recognition Based on Accelerometer and EMG Sensors", IEEE transactions on systems, man, and cybernetics - part a: systems and humans, vol. 41, no. 6 , november 2011.

[10] K.Rama Linga Reddy, G.R. Babu2, Dr. Lal Kishore, "Face Recognition Based on Eigen Features of Multi Scaled Face Components and Artificial Neural Network", International Journal of Security and Its Applications Vol. 5 No. 3, July, 2011.

[11] G. Simion, V. Gui, and M. Otesteanu, "Vision Based Hand Gesture Recognition: A Review", international journal of circuits, systems and signal processing, Issue 4, Volume 6, 2012.

[12] Jyoti S. Bedre, Shubhangi Sapkal, "Comparative Study of Face Recognition Techniques: A Review", Emerging Trends in Computer Science and Information Technology -2012 (ETCSIT2012) Proceedings published in International Journal of Computer Applications ${ }^{\circledR}$ (IJCA).

[13] Siddharth S. Rautaray, Anupam Agrawal, "Real Time Multiple Hand Gesture Recognition System for Human Computer Interaction", I.J. Intelligent Systems and Applications, 2012, 5, 56-64 Published Online May 2012 
in MECS (http://www.mecs-press.org/) DOI: 10.5815/ijisa.2012.05.08

[14] Satonkar Suhas S, Kurhe Ajay B, Dr.Prakash Khanale B, "Face Recognition Using Principal Component Analysis and Linear Discriminant Analysis on Holistic Approach in Facial Images Database", IOSR Journal of Engineering e-ISSN: 2250-3021, p-ISSN: 2278-8719, www.iosrphr.org Vol. 2, Issue 12 (Dec. 2012), \|V4\| PP 15-23.

[15] Aseema Sultana, T Rajapuspha, "Vision Based Gesture Recognition for Alphabetical Hand Gestures Using the SVM Classifier", International Journal of Computer Science \& Engineering Technology (IJCSET), ISSN : 2229-3345, Vol. 3 No. 7 July 2012.

[16] Archana S Archana S. Ghotkar, Gajanan K. Kharate, "Hand Segmentation Techniques to Hand Gesture Recognition for Natural Human Computer Interaction", International Journal of Human Computer Interaction (IJHCI), Volume (3) : Issue (1) : 2012.

[17] Rung-Huei Liang1, Ming Ouhyoung2, “A Real-time Continuous Gesture Recognition System for Sign Language", IEEE International Conference on Automatic Face and Gesture Recognition, pp. 558-567, Japan, 1998.

[18] Shekhar Singh ${ }^{1}$, Akshat Jain ${ }^{2}$, Deepak Kumar ${ }^{3}$, "Recognizing and Interpreting Sign Language Gesture for Human Robot Interaction", International Journal of
Computer Applications (0975 - 8887) Volume 52No.11, August 2012.

[19] Bhavina Patel, Vandana Shah, Ravindra Kshirsagar, "Microcontroller based gesture recognition system for the handicap people", Journal of Engineering Research and Studies, E-ISSN0976-7916.

[20] Raymond Lockton and Andrew W. Fitzgibbon, "Realtime gesture recognition using deterministic boosting", British Machine Vision Conference.

[21] K M Poornima, Ajit Danti, S K Narasimhamurthy, "Wavelet Based Face Recognition using ROIs and kNN", International Journal of Innovations in Engineering and Technology (IJIET), Vol. 3 Issue 2 December 2013.

[22] Simarnjot singh, Navneet kaur, "A Hand recognition system based on SIFT algorithm", October 2013, vol .2 issue 10, www.ijirs.com

[23] Er. Sarbjeet Kaur, Dr. V.K. Banga, "Real-Time Capable System For Hand Gesture Recognition Using Matrix Method In MATLAB For Indian Sign Language", 1st National Conference on Information Technology and Cyber Security/Vol.1/PP 362-367/ITCS13/67.

[24] Arathy.V, Dr.P.Srinivasa Babu, "Recognition of Face Images through the Fusion Approach", International Conference on Engineering Technology and Science(ICETS'14), ISSN (Online) : 2319 - 8753 ISSN (Print) : $2347-6710$ 